\title{
Deep Learning Methods for Cardiovascular Diseases
}

\author{
Yankun Cao ${ }^{1}$, Zhi Liu ${ }^{1,}{ }^{*}$, Pengfei Zhang ${ }^{2}$, Yushuo Zheng ${ }^{3}$, Yongsheng Song ${ }^{4}$, Lizhen Cui ${ }^{5}$ \\ ${ }^{1}$ School of Information Science and Engineering, Shandong University, Qingdao 266237, China \\ ${ }^{2}$ Qilu Hospital, Shandong University, Jinan, 250014, China \\ ${ }^{3}$ High School Attached to Shandong Normal University, Jinan, 250014, China \\ ${ }^{4}$ Kedun Science and Technology Co., Ltd., Laiyang, 265200, China \\ ${ }^{5}$ School of Software, Shandong University, Jinan, 250001, China \\ *Corresponding Author: Zhi Liu (liuzhi@sdu.edu.cn)
}

How to cite this paper: Yankun Cao; Zhi

Liu; Pengfei Zhang; Yushuo Zheng; Yongsheng Song; Lizhen Cui (2019) Deep Learning Methods for Cardiovascular Image. Journal of Artificial Intelligence and Systems, 1, 96-109.

https://doi.org/10.33969/AIS.2019.11006

Received: September 8, 2019

Accepted: October 16, 2019

Published: November 4, 2019

Copyright (C) 2019 by author(s) and Institute of Electronics and Computer.

This work is licensed under the Creative Commons Attribution International License (CC BY 4.0).

http://creativecommons.org/licenses/by/4.0/

(c) (i) Open Access

\begin{abstract}
In the medical field, the analysis and processing of medical images plays an important auxiliary role in the diagnosis of diseases. In recent years, more and more researchers have begun to pay attention to such processing technologies as pattern recognition, classification and segmentation in medical image processing. Cardiovascular disease is one of the most important diseases that endanger human health at present. It is very meaningful to diagnose and treat cardiovascular disease by means of in-depth learning. In order to make deep learning better applied to cardiovascular diseases, this paper first outlines the development and causes of cardiovascular diseases, then describes several theoretical models of deep learning, and then summarizes the application of deep learning in heart image segmentation, classification and other aspects combined with existing technologies. Finally, the future direction of development is prospected.
\end{abstract}

\section{Keywords}

Deep learning, Cardiovascular Diseases, Cardiac imaging

\section{Introduction}

In recent years, the incidence and mortality of coronary heart disease have increased year by year in the world[1]. How to diagnose and prevent coronary heart disease has become an important issue of concern. In the lumen of the coronary arteries, due to the accumulation of fibrosis and calcification, the lumen is narrowed to produce coronary heart disease. The formation of plaque usually takes 15-20 years, and plaque is an important part of the thrombus. Acute cardiovascular events are catastrophic, primarily due to atherosclerotic (AS) plaque rupture and secondary thrombosis. Before the AS plaque ruptures, the AS will have a long period of quiet silence. This has led to difficulties in early screening of high-risk populations and may lead to unnecessary 
over-screening of low-risk groups. Many patients have entered advanced stages of the disease at the first visit, such as ischemic cardiomyopathy and acute coronary syndrome. Even if AS plaques are found, whether or not the lesions require intervention or vulnerability in clinical practice is a challenge that needs to be addressed.

Ischemic cardiomyopathy is the final battlefield of clinical management of coronary heart disease. Although the mortality rate of patients with coronary heart disease began to decline in only 10 years, the incidence of heart failure increased dramatically. The changes of myocardial structure and function and the occurrence of cardiac remodeling are accompanied by malignant arrhythmia, systemic circulation embolism, multiple organ injury and a series of thorny complications. The 5-year survival rate of ischemic cardiomyopathy is only $26-52 \%$, which is similar to the mortality rate of malignant tumors. In addition, the accurate assessment of cardiac remodeling and cardiac function, the range of viable myocardium and myocardial elasticity is of decisive significance for the formulation of a series of interventional strategies, such as revascularization, resynchronization pacing, cardiac transplantation and optimization of drug regimens. In summary, we can see that the clinical management practice of cardiovascular disease, especially coronary heart disease, is facing various problems such as how to reduce the cost of prevention and treatment, how to optimize cost-effectiveness, how to avoid excessive intervention and inadequate patient management, and how to reduce the high readmission rate and mortality. This article will focus on cardiovascular diseases and explore the new vitality of in-depth learning for cardiovascular diseases.

At present, there are many ways to collect medical images, including magnetic resonance imaging (MRI), computed tomography (CT), X-ray imaging (X-rays), ultrasound imaging (Ultrasound imaging), positron emission tomography (PET). Pathological optical microscopy plays an important role in detecting the anatomical and functional information of different body organs for diagnosis and research [2][3][4][5].

In recent years, due to the rapid development of deep learning, people began to use deep learning technology in medical image analysis. At present, deep learning has made great progress in medical disease classification, lesion detection and segmentation and medical image registration[6][7][8][9]. Medical image classification refers to the use of deep learning method. Firstly, the object is pre-classified, and then the classification model is obtained by training. Finally, the classification of medical images is judged according to the model. Medical image segmentation is a preprocessing step for feature extraction and classification, which separates abnormal or special parts in medical images. Traditional image segmentation techniques include edge detection, threshold segmentation, region segmentation, clustering segmentation, etc. Later, watershed technology based on morphology and segmentation technology based on deformation model are introduced. In order to meet the needs of complex image segmentation, more and more people begin to turn their attention to depth learning, and the research of medical image segmentation based on depth learning is flourishing. In medical image registration, two or more images must be spatially aligned so that the same location in 
each image represents the same physical location in the described organ. At first, in image registration, depth learning is used to measure similarity. This similarity measure is called mutual information, which is better than standard measure. Later, CNN in depth learning was used for end-to-end training to generate spatial transformation to minimize the dissimilarity between misaligned images for registration on various medical images.

\section{Deep learning method}

Deep learning is a new field in machine learning research. Its motivation lies in the establishment and simulation of the human brain to analyze and study the neural network, which mimics the human brain mechanism to explain the data [10]. The concept of deep learning is derived from the study of artificial neural networks, and the multi-layer sensor with multi-hidden layer is a deep learning structure. The essence of deep learning is to learn more useful features by building a number of hidden machine learning models and massive training data to improve the accuracy of classification or prediction. In this section, we will introduce several typical models of deep learning.

\subsection{Auto Encoder}

One of the simplest ways to deep learning is to use the characteristics of artificial neural networks. Given a neural network, assume that the input and output are the same, and then train and adjust the parameters; you can get the weight of each layer [11]. Then we can get several different representations of input I (each layer represents a representation), and these representations are features. The automatic encoder is such a neural network that can reproduce the input signal as much as possible. The auto encoder is an unsupervised learning, and its basic block diagram is shown in Figure 1 below.

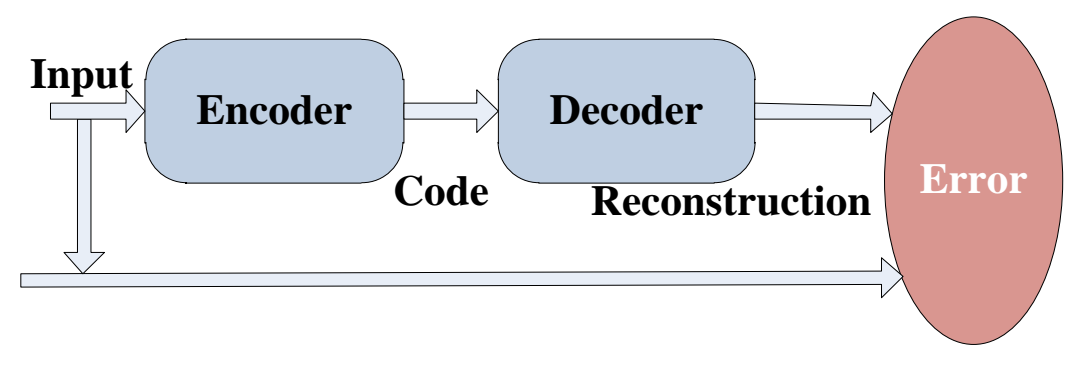

Figure 1. Structure of auto encoder

In order to reconstruct the input signal, the automatic encoder must capture the most important factors that can represent the input data. We define $\mathrm{X}$ is the input. So that the code is

$$
h=W^{T} X,
$$

Where the $\mathrm{W}$ is the weight matrix. That is, by learning to make the output more and more approaching and input. 


\subsection{Sparse Coding}

If we do not strictly require input and output equal, then the input and output relationship can be expressed as

$$
X=\sum_{i=1}^{k} a_{i} \phi_{i}
$$

Where $\mathrm{X}$ is the input, $a_{i}$ is the coefficient, $\phi_{i}$ is the basis. If add $a_{i}$ sparsely limited, then this method is called sparse coding. Sparse coding has been successfully applied to a variety of problems in computer vision and image analysis [12]. Sparse coding algorithm is an unsupervised learning method, which is used to find a set of "super complete" basis vector to more efficient representation of the sample data[13]. The purpose of the sparse coding algorithm is to find a set of base vectors that allow us to represent input vectors as linear combinations of these base vectors. The sparse coding process is divided into two parts, the training phase and the coding phase.

\subsubsection{Training}

The sparse coding training process is an iterative process of finding the optimal solution. We assume that a series of the input signal data is $\mathrm{X}=\left[x_{1}, x_{2}, \ldots, x_{n}\right]$. Each $x_{i}$ represents a sample data. We intend to find a set of basis $\Phi=\left[\phi_{1}, \phi_{2}, \ldots, \phi_{k}\right]$ (That is what we usually say the dictionary) to satisfy

$$
\langle a, \Phi\rangle=\underset{a, \Phi}{\arg \min } \sum_{i=1}^{n}\left\|x_{i}-\sum_{j=1}^{k} a_{i, j} \phi_{j}\right\|^{2}+\lambda \sum_{i=1}^{n} \sum_{j=1}^{k}\left|a_{i, j}\right|
$$

Where $a$ is Sparse coding matrix, $\lambda$ is a parameter of equilibrium reconstruction error and sparseness.

Sparse coding of the iterative process can be divided into two steps:

a) Fixed $\Phi[\mathrm{k}]$ and then continue to adjust $a[\mathrm{k}]$, minimizes the objective function;

b) Fixed $a[\mathrm{k}]$ and adjust $\Phi[\mathrm{k}]$, minimizes the objective function.

Through such an alternating iterative process we can get such a set of dictionaries, can be good to express the input signal $\mathrm{X}$.

\subsubsection{Coding}

Coding stage is very simple. When given a new signal $\mathrm{x}$, we only need to learn from the above dictionary, the sparse expression of $\mathrm{x}$ is to meet the following formula a.

$$
\langle a\rangle=\arg \underset{a}{\min } \sum_{i=1}^{n}\left\|x_{i}-\sum_{j=1}^{k} a_{i, j} \phi_{j}\right\|^{2}+\lambda \sum_{i=1}^{n} \sum_{j=1}^{k}\left|a_{i, j}\right|
$$

\subsection{Restricted Boltzmann Machine (RBM)}

Suppose there is a bipartite graph, each layer of nodes is not connected. Layer is the visual layer, that is, input data layer v. Layer is hidden layer h. If we assume that all nodes are random binary variable nodes and the full probability distribution $\mathrm{p}(\mathrm{v}, \mathrm{h})$ 
satisfies the Boltzmann distribution, we call this model a restricted boltzmann machine (RBM).

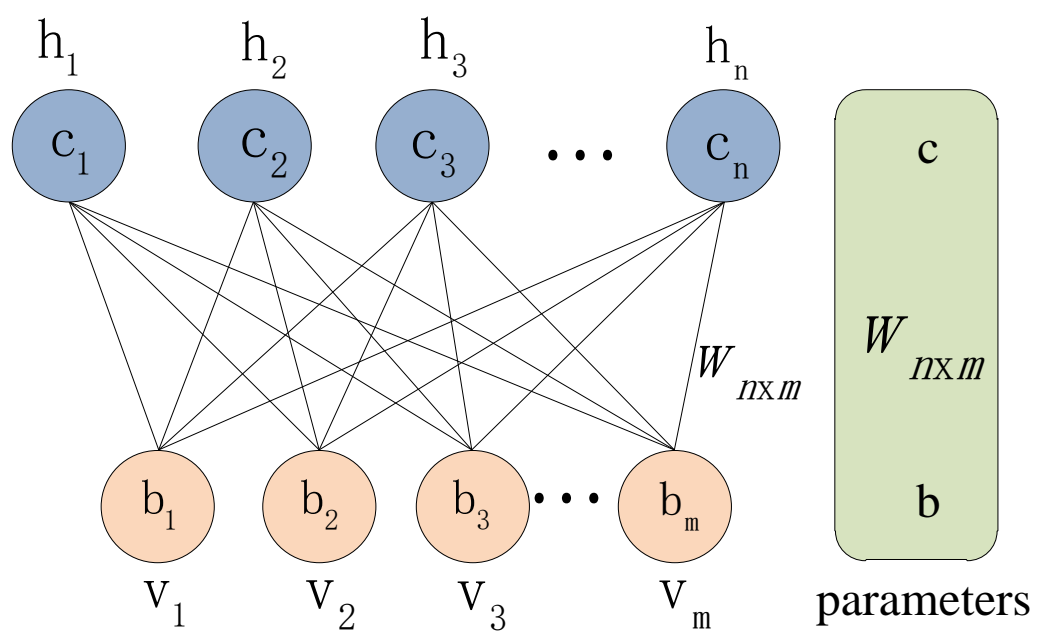

Figure 2. RBM network structure

This RBM network has $n$ hidden nodes, m visible nodes. In the network, the parameter $W_{n \times m}$ is the weight matrix, $b$ is the offset of the visible node, and $c$ is the offset of the hidden node. RBM network is to have these parameters to determine the n-dimensional input samples encoded as m-dimensional feature samples.

Let's take a look at the RBM network training process. First assume that each node value is in the set $\{0,1\}$, that is $\forall \mathrm{i}, j, v_{i} \in\{0,1\}, h_{j} \in\{0,1\}$. A training sample $x=\left(x_{1}, x_{2} \cdots x_{n}\right)$, according to the RBM network can be encoded after the sample $y=\left(y_{1}, y_{2} \cdots y_{m}\right)$. This m-dimensional sample can be viewed as a sample of $\mathrm{m}$ features extracted from n-dimensional input samples.

The probability that the $\mathrm{j}$ characteristic of a hidden node is 1 is

$$
p\left(h_{j}=1 \mid \mathrm{v}\right)=\sigma\left(\sum_{\mathrm{i}=1}^{n} w_{j i} v_{i}+c_{j}\right)
$$

Similarly, on the basis of a given hidden layer, the probability that the i node of the visual layer is 0 or 1 is

$$
p\left(v_{i}=1 \mid h\right)=\sigma\left(\sum_{\mathrm{j}=1}^{\mathrm{m}} \mathrm{w}_{\mathrm{ij}} \mathrm{h}_{\mathrm{j}}+\mathrm{b}_{\mathrm{i}}\right)
$$

Where the $v_{i}$ is the input $x_{i}, h_{j}$ is the $\mathrm{y}_{j}, \sigma(\mathrm{x})=\frac{1}{1+\exp (-\mathrm{x})}$.

The coding process is that first the probability $p\left(h_{j}=1 \mid \mathrm{v}\right)$ is calculated from the value of $\mathrm{v}$ using Equation (5), and then a random number between 0 and 1 is generated. If it is less than $p\left(h_{j}=1 \mid \mathrm{v}\right)$, the value of $\mathrm{h}$ is 1 , otherwise it is 0 . Similarly, here is a coded sample y, the decoding process is to first use the formula (7) according to the value of $h$ 
to calculate the probability $p\left(v_{i}=1 \mid \mathrm{h}\right)$, where the value of $h_{j}$ is the value of $\mathrm{y}_{j}$. And then randomly generate a random number between 0 and 1 . If it is less than $p\left(v_{i}=1 \mid \mathrm{h}\right)$, the value of $v_{i}$ is 1 otherwise is 0 .

\subsection{Deep Belief Networks}

From the above RBM network we can get DBN. DBN (Deep Belief Net) is to increase the hidden layer in RBM, in the vicinity of the visual layer to use the Bayesian belief network, where the node is not connected to the node is not connected, away from the visual layer part of the use of RBM.

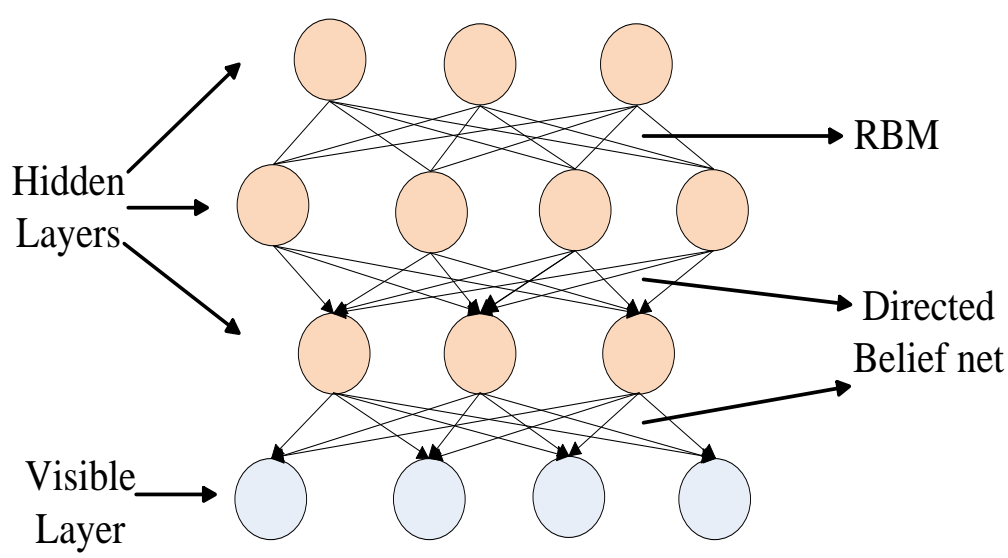

Figure 3. DBN structure

The classic DBN network structure is composed of several layers of RBM and a layer of BP composed of a deep neural network, the structure shown Fig.4.

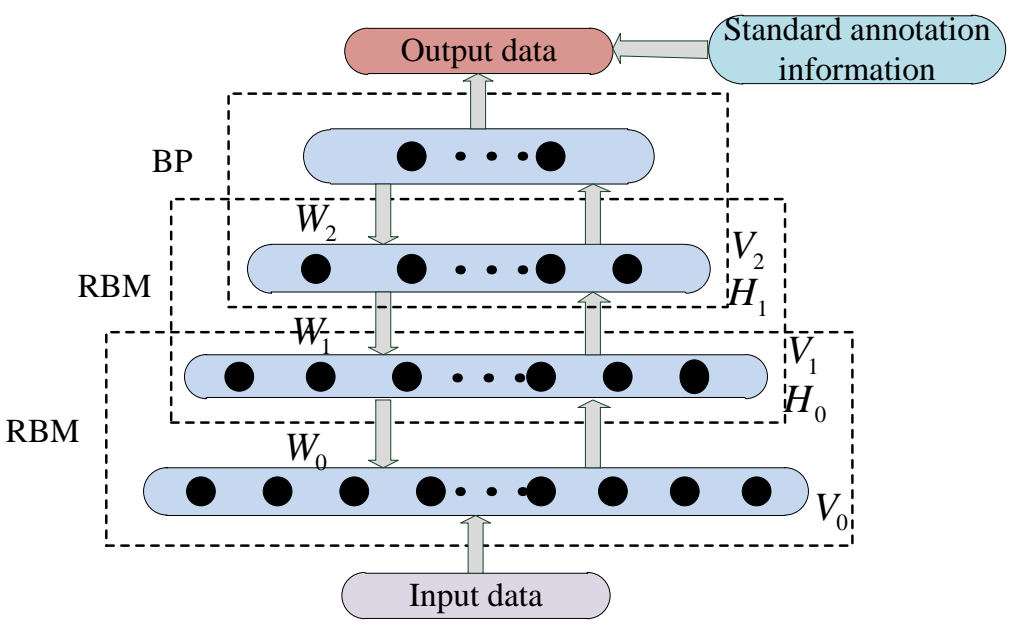

Figure 4. Classic DBN network structure

DBN in the training model is mainly divided into two steps: 
a) Respectively, unsupervised training of each layer of RBM network, to ensure that the eigenvector mapping to different feature space as much as possible to retain the characteristics of information.

b) The BP network is set up at the last level of the DBN, accepting the output feature vector of RBM as its input feature vector, and supervising the training entity relation classifier. Moreover, each RBM network can only ensure that the weights in the self-layer are optimal to the eigenvector mapping, rather than the eigenvector mapping of the whole DBN. So the back-propagation network also spread the error message from top to bottom to each layer RBM, fine-tuning the entire DBN network. The process of RBM network training model can be regarded as the initialization of a deep BP network weight parameter, which makes DBN overcome the shortcomings of BP network due to random initialization of weight parameters and easy to fall into local optimum and long training time.

\subsection{Convolutional Neural Networks}

Convolution neural network is a kind of artificial neural network, which has become a hotspot in the field of speech analysis and image recognition. One of its salient features is weight sharing. This greatly reduces the complexity of the network model, reducing the number of weights. The fMRI image is a three-dimensional image, which can be directly input as a CNN, thus avoiding the complex feature extraction and data reconstruction process in the traditional recognition algorithm. In the CNN, a small portion of the image is the lowest input of the hierarchy, and the information is then transferred to different layers, each of which passes through a digital filter to obtain the most significant feature of the observed data. This method can obtain significant characteristics of the observed data for translation, scaling and rotation. CNN network structure is shown in Fig.5.

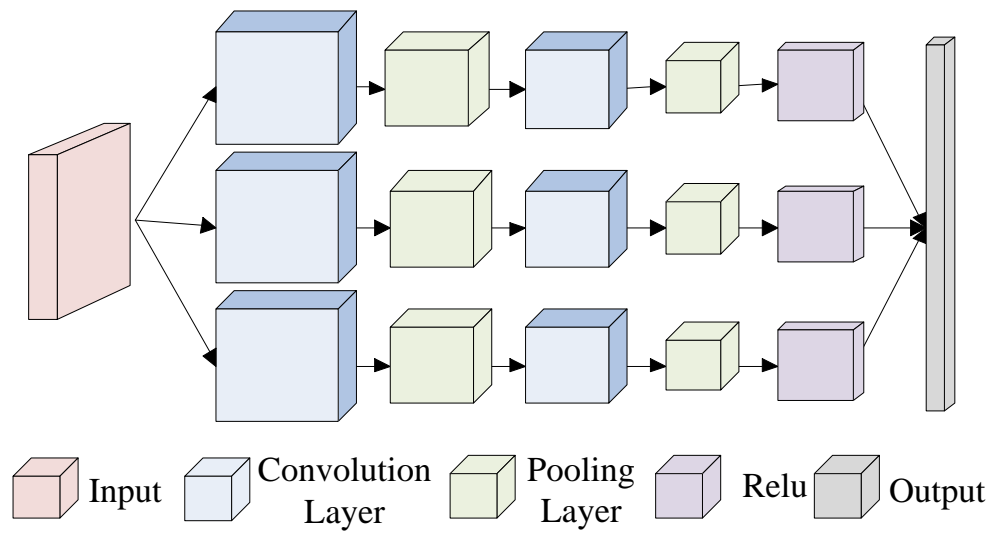

Figure 5. CNN network structure

Convolution neural network training algorithm and the traditional BP algorithm is almost, mainly including four steps, the four is divided into two stages:

The first stage, forward propagation phase: 
a) Take a sample from the sample set $\left(X, Y_{p}\right)$ and enter $\mathrm{X}$ into the network.

b) Calculate the corresponding actual output $O_{p}$.

At this stage, the information is transferred from the input layer to the output layer via stepwise transformation. This process is also the network in the completion of training after the normal operation of the implementation process. In the process, the network executes the input with the weight matrix of each layer to multiply, resulting in the final output.

$$
O_{p}=F_{n}\left(\cdots\left(F_{2}\left(F_{1}\left(X_{p} \mathrm{~W}^{(1)}\right) W^{2}\right) \cdots\right) W^{(n)}\right)
$$

The second stage, the backward propagation phase

a) Calculate the difference between the actual output $O_{p}$ and the corresponding ideal output $Y_{p}$.

b) Adjust the weight matrix by inverse propagation error.

\section{Deep Learning Pipeline for Cardiac Ultrasound Imaging}

This section describes end-to-end pipelines for heart disease, based on the three main parts shown in Figure 6. The whole process is divided into data acquisition and preprocessing, network selection, and training and evaluation of network performance. Below is a brief introduction to these three parts.

\subsection{Data acquisition and preprocessing}

Medical images are generally collected in DICOM format, which needs to be converted to an image format before image processing. There are a lot of tools that can be converted directly, for example, it can also be read by the dicomread function in MATLAB. After converting an image to JPG or PNG format, you first need to preprocess the image, such as removing poor quality images, denoising, and tagging. Make the corresponding image into a data set before you can use it for the next step.

\subsection{Network selection}

With the breakthrough of GPU hardware technology and the expanding scale of labeled data in ImageNet, deep learning technology has been promoted, and it has also made a breakthrough in image recognition. In 2012, Hinton and his student Alex Krizhevsky [14] proposed the deep convolution neural network AlexNet. With the help of stochastic gradient descent (SGD) and Dropout optimization techniques, Hinton won the ILSVRC championship in 2012, and achieved a good result of top-5 error rate of $15.3 \%$. Since then, a large number of researchers have begun to enter this field, and in-depth learning has become irremediable, which leads to explosive research. In 2014, Christian Szeged et al. [15] designed Google LeNet and Karen Simonyan et al. [16] designed VGGNet, which made the network structure of in-depth learning break through in width and depth, and won the championship and the third place respectively in ILSVRC 2014. In 2015, Kaiming He et al. [17] made a breakthrough in the number of layers of neural networks, 
designed 152 layers of ResNet, and the error rate of ResNet also fell to 3.6\% which surpassed the human level, which shocked the academic community. Different networks have different advantages and disadvantages, and each has its own applicable environment. Therefore, different networks can be selected according to different tasks to train their own data and achieve their goals.

\subsection{Training and evaluation of network performance}

When deciding on a cardiac ultrasound task, first select the most suitable network, then adjust the parameters based on the network or change the corresponding layer settings of the network as needed to optimize performance. Finally, you can test the network on the test set, and if the effect is equally good then you can finally take this network to perform this task.

Currently, researchers in deep learning have developed a variety of learning platforms, such as Caffe[18], DarkNet, Tensorflow[19], PyTorch[20], Keras, MxNet, Theano, CNTK, etc. Each deep learning platform has its own characteristics and has its own shortcomings. Different learning platforms can be selected according to different networks.

\section{Application of Deep Learning in Cardiac Image}

With the continuous development of artificial intelligence and deep learning, this technology has shown superior advantages in image processing. Due to the complexity of medical images, applying this method to medical image analysis and processing and analyzing medical images using deep learning will greatly reduce the burden on doctors and improve the efficiency of doctors in disease diagnosis. At present, there are many more and more researchers who use deep learning for cardiovascular diseases, such as segmentation, classification and registration. The specific research contents are shown in the following table 1-2:

Table 1. Heart image segmentation using deep learning

\begin{tabular}{cccccc}
\hline Author & Year & Dataset & $\begin{array}{c}\text { Segmentation } \\
\text { content }\end{array}$ & Method & Dice \\
\hline $\begin{array}{c}\text { Rudra P. K. Poudel } \\
\text { et al[21] }\end{array}$ & 2016 & PRETERM & Heart & RFCN & 0.935 \\
\hline Wenjia Bai et al[22] & 2017 & $\begin{array}{c}\text { UK Biobank } \\
\text { study }\end{array}$ & $\begin{array}{c}\text { Short Axis } \\
\text { Heart }\end{array}$ & $\begin{array}{c}\text { Semi- } \\
\text { supervised } \\
\text { Learning }\end{array}$ & 0.92 \\
\hline $\begin{array}{c}\text { Baumgartner, } \\
\text { Christian F et al[23] }\end{array}$ & 2017 & $\begin{array}{c}\text { ACDC } \\
\text { challenge }\end{array}$ & $\begin{array}{c}\text { short-axis } \\
\text { cardiac }\end{array}$ & $\begin{array}{c}\text { FCN } \\
\text { U-Net }\end{array}$ & 0.950 \\
\hline $\begin{array}{c}\text { Ozan Oktay et al[24] } \\
\text { 2017 }\end{array}$ & $\begin{array}{c}\text { UK Digital } \\
\text { Heart Project }\end{array}$ & $\begin{array}{c}\text { short-axis } \\
\text { cardiac }\end{array}$ & ACNN & 0.939 \\
\hline $\begin{array}{c}\text { Yakun Chang et } \\
\text { al[25] }\end{array}$ & 2018 & ACDC & short-axis & FCN & 0.90 \\
\hline
\end{tabular}


Table 2. Heart image classification using deep learning

\begin{tabular}{|c|c|c|c|c|c|}
\hline Author & Year & Dataset & $\begin{array}{c}\text { Classification } \\
\text { content }\end{array}$ & Method & $\begin{array}{c}\text { Accura } \\
\text { cy } \\
\end{array}$ \\
\hline $\begin{array}{c}\text { Lasya PriyaKotu } \\
\text { et al[26] }\end{array}$ & 2015 & $\begin{array}{c}\text { Author } \\
\text { self-made } \\
\text { data }\end{array}$ & $\begin{array}{l}\text { the risk of } \\
\text { arrhythmias }\end{array}$ & $\mathrm{k}-\mathrm{NN}$ & 0.94 \\
\hline Yu Gan et al[27] & 2016 & $\begin{array}{c}\text { Author } \\
\text { self-made } \\
\text { data }\end{array}$ & $\begin{array}{l}\text { human atrial } \\
\text { tissue }\end{array}$ & $\begin{array}{l}\text { region- } \\
\text { based }\end{array}$ & 0.8041 \\
\hline $\begin{array}{c}\text { Houman } \\
\text { Ghaemmaghami } \\
\text { et al[28] }\end{array}$ & 2017 & $\begin{array}{c}\text { Author } \\
\text { self-made } \\
\text { data }\end{array}$ & heart-sound & TDNN & 0.95 \\
\hline $\begin{array}{c}\text { XiaohongGao et } \\
\text { al[29] }\end{array}$ & 2016 & $\begin{array}{c}\text { Tsinghua } \\
\text { University } \\
\text { Hospital }\end{array}$ & $\begin{array}{c}\text { viewpoint of } \\
\text { echocardiography }\end{array}$ & $\begin{array}{l}\text { improved } \\
\text { CNN }\end{array}$ & 0.921 \\
\hline $\begin{array}{c}\text { Ali Madani et } \\
\text { al[30] }\end{array}$ & 2018 & $\begin{array}{c}\text { Author } \\
\text { self-made } \\
\text { data }\end{array}$ & $\begin{array}{c}\text { view of } \\
\text { echocardiograms }\end{array}$ & CNN & 0.978 \\
\hline
\end{tabular}

In addition, there are other attempts, such as Fabian Isensee et al. for automated heart disease assessment on cine-MRI through time series segmentation and domain-specific features[31]. Marc-Michel Rohé et al. use shape matching to learn to apply deformation to heart registration problems between patients [32]. Zhifan Gao et al. employed a linear elasticity model of the carotid artery wall, and converted it into the state space equation in 2017 [33]. Xiantong Zhen et al. propose a new, general framework for direct and simultaneous four chamber volume estimation in 2017[34]. Shen Zhao et al. developed a state-space framework to sequentially segment the carotid IM borders in each image throughout the cardiac cycle in 2018 [35]. Zhifan Gao et al. developed an implicit framework (UE-LUPI) using the deep neural network to reconstruct the strain field in quasistatic ultrasound elastography in 2019 [36]. Lin Xu et al. were to evaluate the value of multi-directional strain parameters derived from three-dimensional (3D) speckle tracking echocardiography (STE) for predicting left ventricular (LV) [37]. Nowadays, deep learning technology has been widely used in medical diagnosis, not only cardiac ultrasound images but also more medical applications waiting to be explored[38][39][40] .

\section{Conclusions and Future Research}

This paper focuses on the pipeline of deep learning cardiac ultrasound imaging. First of all, several models of deep learning are introduced, and the basic principles and architectures of several depth learning methods are briefly explained. Then the development status of cardiac ultrasound images and the application of deep learning are introduced. Deep reading has now become an important tool for heart disease analysis by 
reading a large amount of literature. However, there are still many shortcomings, such as insufficient data, resulting in low accuracy, and currently only in theory, it is difficult to apply. There are many things worth studying in the future, such as combining deep learning with 5G technology to create a new medical model for smart medical and remote diagnosis.

\section{Acknowledgements}

This work was supported in part by the National Key Research and Development Program of China under Grant 2018YFC0831006-3 and in part by the Key Research and Development Plan of Shandong Province under Grant 2017CXGC 1503 and Grant 2018GSF118228, and the fundamental research funds of Shandong University 2018JC009.

\section{Conflicts of Interest}

A Conflict of Interest Statement needs to be included at the end of the manuscript before the references. Here, the authors need to declare whether or not the submitted work was carried out in the presence of any personal, professional or financial relationships that could potentially be construed as a conflict of interest.

\section{References}

[1] World health statistics 2018: monitoring health for the SDGs, sustainable development goals. Geneva: World Health Organization; 2018. Licence: CC BY-NC-SA 3.0 IGO

[2] Wong K K L, Tu J, Sun Z, et al. Methods in research and development of biomedical devices[M]. WORLD SCIENTIFIC, 2013.

[3] MacNeill, B. D. Intravascular Modalities for Detection of Vulnerable Plaque: Current Status [J]. Arteriosclerosis, Thrombosis, and Vascular Biology, 2003, 23(8):1333-1342.

[4] Kips J G, Segers P , Bortel L M V . Identifying the vulnerable plaque: A review of invasive and non-invasive imaging modalities[J]. Artery Research, 2008, 2(1):21-34.

[5] Jaffer F A, Libby P , Weissleder R . Optical and Multimodality Molecular Imaging: Insights Into Atherosclerosis [J]. Arteriosclerosis, Thrombosis, and Vascular Biology, 2009, 29(7):1017-1024.

[6] Anthimopoulos M, Christodoulidis S, Ebner L, et al. Lung pattern classification for interstitial lung diseases using a deep convolutional neural network[J]. IEEE transactions on medical imaging, 2016, 35(5): 1207-1216.

[7] Miao S, Wang Z J, Liao R. A convolutional neural network approach for $2 \mathrm{~d} / 3 \mathrm{~d}$ medical image registration[J]. CoRR abs/1507.07505, 2015.

[8] Vardhana M, Arunkumar N, Lasrado S, et al. Convolutional neural network for bio-medical image segmentation with hardware acceleration[J]. Cognitive Systems Research, 2018, 50: 10-14. 
[9] K. J. Xia, H. S. Yin, J. Q. Wang, “A novel improved deep convolutional neural network model for medical image fusion,” Cluster Computing, 2018, 3:1-13.

[10] Lecun Y, Bengio Y, Hinton G. Deep learning[J]. Nature, 2015, 521(7553):436-444.

[11] Xiao Z, Huang R, Ding Y, et al. A deep learning-based segmentation method for brain tumor in MR images[C]// IEEE, International Conference on Computational Advances in Bio and Medical Sciences. IEEE, 2017:1-6.

[12] Jiang Z, Lin Z, Davis L S. Label Consistent K-SVD: Learning A Discriminative Dictionary for Recognition[J]. IEEE Transactions on Pattern Analysis \& Machine Intelligence, 2013, 35(11):2651-64.

[13]Zheng $\mathrm{M}$, Bu J, Chen C, et al. Graph Regularized Sparse Coding for Image Representation[J]. IEEE Transactions on Image Processing A Publication of the IEEE Signal Processing Society, 2011, 20(5):1327.

[14] Krizhevsky A, Sutskever I, Hinton G E. Imagenet classification with deep convolutional neural networks[C]. Advances in neural information processing systems, 2012: 1097-1105.

[15] Szegedy C, Liu W, Jia Y, et al. Going deeper with convolutions[C]. IEEE Conference on Computer Vision and Pattern Recognition, 2015: 1-9.

[16] Simonyan K, Zisserman A. Very deep convolutional networks for large-scale image recognition[J]. arXiv preprint arXiv: 1409.1556, 2014.

[17] He K, Zhang X, Ren S, et al. Deep residual learning for image recognition[C]. Proceedings of the IEEE conference on computer vision and pattern recognition, 2016: 770-778.

[18] Caffe [Online]. Available: http://caffe.berkeleyvision.org/.(Accessed on 24 May 2016)

[19] Tensorflow [Online]. Available: https://tensorflow.org/. (Accessed on 2016)

[20] Pytorch [Online]. Available: https://pytorch.org/. (Accessed on 2017)

[21] Poudel R P K, Lamata P, Montana G. Recurrent fully convolutional neural networks for multi-slice MRI cardiac segmentation[M]//Reconstruction, segmentation, and analysis of medical images. Springer, Cham, 2016: 83-94.

[22] Bai W, Oktay O, Sinclair M, et al. Semi-supervised learning for network-based cardiac MR image segmentation[C]//International Conference on Medical Image Computing and Computer-Assisted Intervention. Springer, Cham, 2017: 253-260.

[23] Baumgartner C F, Koch L M, Pollefeys M, et al. An exploration of 2D and 3D deep learning techniques for cardiac MR image segmentation[C]//nternational Workshop on Statistical Atlases and Computational Models of the Heart. Springer, Cham, 2017: 111-119.

[24] Oktay O, Ferrante E, Kamnitsas K, et al. Anatomically constrained neural networks (ACNNs): application to cardiac image enhancement and segmentation[J]. IEEE transactions on medical imaging, 2017, 37(2): 384-395.

[25]Chang Y, Song B, Jung C, et al. Automatic Segmentation and Cardiopathy Classification in Cardiac Mri Images Based on Deep Neural Networks[C]//2018 IEEE 
International Conference on Acoustics, Speech and Signal Processing (ICASSP). IEEE, 2018: 1020-1024.

[26] Kotu L P, Engan K, Borhani R, et al. Cardiac magnetic resonance image-based classification of the risk of arrhythmias in post-myocardial infarction patients[J]. Artificial intelligence in medicine, 2015, 64(3): 205-215.

[27]Gan Y, Tsay D, Amir S B, et al. Automated classification of optical coherence tomography images of human atrial tissue[J]. Journal of biomedical optics, 2016, 21(10): 101407.

[28] Ghaemmaghami H, Hussain N, Tran K, et al. Automatic segmentation and classification of cardiac cycles using deep learning and a wireless electronic stethoscope[C]//2017 IEEE Life Sciences Conference (LSC). IEEE, 2017: 210-213.

[29] Gao X, Li W, Loomes M, et al. A fused deep learning architecture for viewpoint classification of echocardiography[J]. Information Fusion, 2017, 36: 103-113.

[30] Madani A, Arnaout R, Mofrad M, et al. Fast and accurate view classification of echocardiograms using deep learning[J]. NPJ digital medicine, 2018, 1(1): 6.

[31] Isensee F, Jaeger P F, Full P M, et al. Automatic cardiac disease assessment on cine-MRI via time-series segmentation and domain specific features[C]//International workshop on statistical atlases and computational models of the heart. Springer, Cham, 2017: 120-129.

[32] Rohé M M, Datar M, Heimann T, et al. SVF-Net: Learning deformable image registration using shape matching[C]//International Conference on Medical Image Computing and Computer-Assisted Intervention. Springer, Cham, 2017: 266-274.

[33] Gao Z, Xiong H, Liu X, et al. Robust estimation of carotid artery wall motion using the elasticity-based state-space approach[J]. Medical image analysis, 2017, 37: 1-21.

[34] Zhen X, Zhang H, Islam A, et al. Direct and simultaneous estimation of cardiac four chamber volumes by multioutput sparse regression[J]. Medical image analysis, 2017, 36: 184-196.

[35] Gao Z Zhao S, Gao Z, Zhang H, et al. Robust Segmentation of Intima-Media Borders With Different Morphologies and Dynamics During the Cardiac Cycle[J]. IEEE journal of biomedical and health informatics, 2017, 22(5): 1571-1582.

[36] Gao Z, Wu S, Liu Z, et al. Learning the implicit strain reconstruction in ultrasound elastography using privileged information[J]. Medical image analysis, 2019, 58: 101534.

[37] Xu L, Huang X, Ma J, et al. Value of three-dimensional strain parameters for predicting left ventricular remodeling after ST-elevation myocardial infarction[J]. The International Journal of Cardiovascular Imaging, 2017, 33(5):663-673.

[38] Automatic microstructural characterization and classification using artificial intelligence techniques on ultrasound signals[J]. Expert Systems with Applications, 2013, 40(8):3096-3105.

[39] Khamparia A, Saini G, Gupta D, et al. Seasonal Crops Disease Prediction and Classification Using Deep Convolutional Encoder Network[J]. Circuits, Systems, and Signal Processing, 2019(May). 
[40] Albuquerque V H C D, Nunes T M, Pereira D R, et al. Robust automated cardiac arrhythmia detection in ECG beat signals[J]. Neural Computing \& Applications, 2018, 29(3):1-15. 